\title{
Desigualdades sociales en los tiempos de espera para la consulta médica en Argentina*
}

\section{Social Inequalities in Waiting Times for Medical Consultation in Argentina}

\section{Desigualdades em tempos de espera para consulta médica na Argentina}

Fecha de recepción: 18-02-16 Fecha de aceptación: 14-04-16 Disponible en línea: 03-05-16 doi:10.11144/Javeriana.rgyps15-30.dste

Cómo citar este artículo:

Ballesteros MS. Desigualdades sociales en los tiempos de espera para la consulta médica en Argentina. Rev. Gerenc. Polit. Salud. 2016; 15(30): 234-250. http://dx.doi.org/10.11144/Javeriana.rgyps15-30.dste

\section{Matías Salvador Ballesteros **}

Artículo de investigación. Resume parte de los resultados de la tesis de doctorado de Matías Salvador Ballesteros titulada "Heterogeneidad y segmentación del sistema sanitario argentino y desigualdad social en el acceso a los servicios de salud: un análisis a partir de la integración de fuentes estadísticas nacionales". Esta investigación fue financiada por el Consejo Nacional de Investigaciones Científcas y Técnicas (Conicet) mediante una beca doctoral tipo I y II (4/2011-3/2016) otorgada a Matías Salvador Ballesteros.

** Lic. en Sociología y doctor en Ciencias Sociales por la Facultad de Ciencias Sociales (FSOC) de la Universidad de Buenos Aires (UBA), Argentina. Becario del Consejo Nacional de Investigaciones Científicas y Técnicas (Conicet) con sede en el Instituto de Investigaciones Gino Germani (IIGG) de la UBA. Correo electrónico: matiballesteros@ yahoo.com.ar. Correspondencia: Instituto de Investigaciones Gino Germani, Uriburu 950, Ciudad Autónoma de Buenos Aires, Argentina. 


\section{Resumo}

Contexto: los tiempos de espera para la atención son una de las principales barreras en el acceso a los servicios de salud en Argentina. Objetivo: analizar los tiempos diferenciales que la población tuvo que aguardar en la sala de espera y entre la solicitud de un turno y la atención con un médico especialista, según sus características socioeconómicas, demográficas, el tipo de cobertura de salud y el lugar de residencia. Método: cuantitativo, basado en el análisis de datos secundarios. Se utilizó la Encuesta de la Deuda Social Argentina del año 2013, realizada por el Observatorio de la Deuda Social Argentina de la Universidad Católica Argentina. Resultados: los tiempos de espera para consultar con un médico estuvieron estratificados socialmente, según las características socioeconómicas, tipo de cobertura y lugar de residencia de la población. No existieron diferencias significativas según el sexo y entre los grupos etarios.

Palavras-chave: accesibilidad a los servicios de salud; atención médica; barreras; inequidad social; tiempo de espera; Argentina

\section{Abstract}

Context: The waiting times for medical attention constitutes one of the main barriers to access to the Healthcare System in Argentina. Objective: Analyse different times that people have to keep in the waiting room and between the request for an appointment and the medical attention with a specialist according to their socio-economic and demographic characteristics, the type of health coverage and place of residence. Method: quantitative, based on the analysis of secondary data. It was implemented in the Survey of "La Deuda Social Argentina" of the year 2013, conducted by the "Observatorio de la Deuda Social Argentina (Universidad Católica Argenitna)". Results: The waiting time to see a doctor it's socially stratified according to socioeconomic characteristics, type of medical coverage and place of residence of the population. There are no significant differences between gender and across age groups.

Keywords: health service accessibility; medical care; barriers; social inequality; waiting time; Argentina

\section{Resumen}

Contexto: os tempos de espera para os cuidados são um dos principais obstáculos no acesso aos serviços de saúde na Argentina. Objetivo: analisar os tempos diferenciais que as pessoas tiveram que esperar na sala de espera e entre o pedido para um compromisso e cuidado com um médico especialista, de acordo com suas características demográficas, socioeconômicas, tipo de cobertura de saúde e residência. Método: quantitativo, com base na análise de dados secundários. Foi utilizada a pesquisa "Encuesta de la Deuda Social Argentina" 2013, realizada pelo "Observatorio de la Deuda Social Argentina (Universidad Católica Argentina)". Resultado: os tempos de espera para ver um médico estiveram socialmente estratificados de acordo com as características socioeconômicas, tipo de cobertura e residência da população. Não houve diferenças significativas por sexo e grupos etários.

Palabras clave: acessibilidade a serviços de saúde; atendimento médico; barreiras; iniquidade social; tempo de espera; Argentina 


\section{Introducción}

El sistema de salud argentino está dividido en tres subsectores: público, de la seguridad social y privado. Se asienta sobre el subsector público, que brinda una cobertura a todos los habitantes del país, aunque sus principales usuarios son los sectores de bajos recursos. Debido a la combinación entre un sistema fuertemente descentralizado en los gobiernos provinciales y municipales y las fuertes desigualdades de recursos entre las distintas jurisdicciones, existen grandes diferencias en los servicios del subsector público según la localización geográfica (1-5). Un segundo subsector es el de la seguridad social, compuesto por instituciones denominadas obras sociales. Este se encarga de la cobertura de los asalariados y trabajadores independientes registrados, los jubilados y pensionados, $\mathrm{y}$ sus familias. Entre las obras sociales existe una gran diversidad en la cantidad de afiliados, recursos por afiliados y de estructura poblacional en cuanto a edad y sexo. Además, algunas obras sociales tienen planes diferenciales, lo cual genera desigualdades en las características de la cobertura entre afiliados a una misma obra social $(3,6,7)$. Por último, el subsector privado está compuesto por empresas de seguros privados de salud (prepagas), prestadores y profesionales independientes. En cuanto a las empresas de medicina prepaga, si bien existe una gran heterogeneidad en la cantidad de afiliados y de recursos por afiliado, estas cubren principalmente a la población de mayores ingresos y más joven (con menos riesgo de enfermarse) y se concentran en los grandes centros urbanos (principalmente en el Área Metropolitana de Buenos Aires) (7-9).

Lo anterior hace que el sistema de salud se encuentre segmentado (conviven distintos subsectores de aseguramiento), fragmen236 tado (sin articulación entre los distintos subsectores e incluso en el interior de ellos), superpuesto y con características y calidades de atención heterogéneas (3,5-7). Las características del acceso a los servicios están fuertemente relacionadas con el tipo de cobertura (privada, de la seguridad social o pública) que los individuos y los grupos familiares poseen, que a la vez depende de su inserción en el mercado de trabajo y su capacidad de pago (voluntaria o por aportes salariales) $(5,10)$.

Una de las dimensiones en que se observan las diferencias en las características del acceso a los servicios de salud es en los tiempos de espera para la atención. Retomando a Frenk (11: 443-6), entendemos que el acceso es una función de los obstáculos generados por la organización de los servicios de salud y los recursos o capacidades para superarlos de la población (el autor lo denomina “poder de utilización”), teniendo en cuenta la disponibilidad física de recursos de salud en una zona determinada. La relación entre los obstáculos generados por los servicios de salud para la búsqueda y obtención de atención y las capacidades o recursos de la población se da en tres dimensiones: 1) la ecológica (relaciona la ubicación geográfica de los lugares de atención con el tiempo y la capacidad de transporte de los usuarios); 2) la financiera (vincula los costos totales para poder utilizar los servicios con los recursos financieros de la población); y 3) la organizacional (relaciona las dificultades organizativas para el contacto inicial con los servicios - como las demoras en otorgar turnos-y las demoras en la sala de espera o para obtener los servicios de laboratorio, con la disponibilidad de tiempo y la tolerancia a las demoras). Es por eso que un aumento (o una disminución) de los tiempos de demora para acceder a la atención dan cuenta de un aumento (o una disminución) de los obstáculos para acceder a los servicios. Pero en términos de accesibilidad solo cobra sig- 
nificado cuando se lo compara con el tiempo disponible de la población.

Por su parte, cabe destacar que las demoras en la asignación de turnos o en una sala de espera pueden hacer que se agrave el motivo que originó la consulta de los usuarios (1219) y, en algunos casos extremos, que estos busquen otras alternativas para resolver las necesidades de salud o abandonen los tratamientos $(20,21)$. Es por esto que las demoras en la atención también dan cuenta de la calidad de los servicios e inciden en la satisfacción de los usuarios, además de que la atención en el momento y lugar en que se la necesita hace a las características del acceso (22-26).

En este artículo analizamos los obstáculos organizacionales diferenciales que el sistema de salud genera según las características de la población (nivel educativo, nivel de ingresos, tipo de cobertura de salud, sexo, grupo etario y lugar de residencia) que necesita consultar con un médico. Particularmente, analizamos el tiempo de demora en la sala de espera en la última consulta médica y los días de espera luego de solicitar un turno con un especialista entre la población de dieciocho años y más residente en medianos y grandes aglomerados de Argentina.

Destacamos la importancia de analizar esta problemática ya que son múltiples los antecedentes que dan cuenta de que los tiempos de espera (27-33) y la obtención de turnos $(30,31,34)$ son algunos de los principales impedimentos para que la población pueda acceder a los servicios de salud en Argentina. Asimismo, examinamos la forma diferencial en que el sistema de salud genera obstáculos para acceder a los servicios según las características de la población que requiere utilizarlos.

\section{Materiales y métodos}

La metodología del artículo es cuantitativa y está basada en el análisis de datos secundarios. Tomamos como fuente de información la "Encuesta de la Deuda Social Argentina" (EDSA), desarrollada por el Observatorio de la Deuda Social Argentina (ODSA) de la Universidad Católica Argentina (UCA) del año 2013. La EDSA se viene realizando anualmente desde el año 2004, pero a partir del 2010 aumentó el tamaño muestral y la cobertura geográfica. Se realiza a partir de un diseño muestral polietápico, que permite realizar estimaciones de los hogares urbanos de medianos y grandes aglomerados del país. En una primera etapa se selecciona un conjunto de conglomerados que es necesario incluir dentro de la muestra y luego se estratifican los radios censales de cada aglomerado a partir de los niveles de educación de los jefes de hogar registrados en Censo Nacional de Población y Vivienda del año 2001. En cada conglomerado se seleccionan puntos muestras de distintos estratos. Por cada punto muestra se seleccionan seis hogares y dentro de cada hogar se encuestó a un individuo de dieciocho años o más (35). La muestra final del año 2013, con la que trabajamos para el presente artículo, quedó conformada por 5689 hogares (36). Dado que se trató de un muestreo probabilístico estratificado no proporcional, se desarrollaron factores de ponderación para ajustar los resultados obtenidos a los parámetros poblacionales. Los datos trabajados en este artículo utilizan estos factores de ponderación, sin expandir los resultados al total de la población.

Entre las distintas temáticas que aborda la EDSA, hay una batería de preguntas sobre la problemática del acceso y los obstáculos que el sistema le genera a la población para utilizar los servicios de salud. En este artículo trabajamos con dos de ellas. Una releva el tiempo que la población debió aguardar en la 
sala de espera en la última consulta médica. Específicamente se preguntó si en la última consulta médica “¿debió esperar más de una hora para que lo atiendan?”. La otra releva los días de espera entre la solicitud del turno con un especialista médico y la atención efectiva. Específicamente se le consultó a la población: "La última vez que pidió un turno con un médico especialista ¿Cuantos días tuvo que esperar para ser atendido?".

Para la elaboración del artículo accedimos a la base de datos, al cuestionario, a documentos metodológicos y entrevistamos a investigadores que fueron parte de la realización de la encuesta. El análisis fue realizado a partir del paquete estadístico spss 17.0. Comenzamos examinando la relación bivariada entre las variables dependientes con variables de la estructura social (nivel educativo y quintil de ingreso per cápita del hogar), demográficas (sexo y grupo etario), el tipo de cobertura de salud y el tipo de aglomerado en el que reside la población. Luego, incluimos las variables significativamente relacionadas (trabajando con un nivel de confianza del 95\%) en una regresión logística paso por paso. Esta es una herramienta que se utiliza para construir modelos de análisis multivariado, que permiten analizar la relación de varias variables independientes con una dependiente dicotómica. A partir de la regresión logística podemos saber cuál es el efecto de una variable independiente sobre la dependiente, una vez controlado el resto (37).

Cabe destacar, con relación a la variable "aglomerado de residencia", que la encuesta permite realizar estimaciones para la Ciudad de Buenos Aires, el Conurbano Bonaerense,

1 Se excluyeron las consultas médicas realizadas por servicio de urgencia o emergencia. En el cuestionario no se incluyeron preguntas que permitan diferenciar el tipo de especialidad de la consulta médica. otras áreas metropolitanas ${ }^{2}$ y el resto urbano del interior. ${ }^{3}$ La Ciudad de Buenos Aires es una jurisdicción autónoma y el Conurbano Bonaerense es un territorio contiguo a la Ciudad de Buenos Aires que se encuentra en la jurisdicción de la Provincia de Buenos Aires. En cambio, las categorías otras “áreas metropolitanas" y "resto urbano del interior" contienen ciudades de distintas jurisdicciones del país. Destacamos por lo anterior que las categorías Ciudad de Buenos Aires y Conurbano Bonaerense hacen referencia a delimitaciones geográficas continuas bajo una jurisdicción política (a su vez, en el interior del conurbano bonaerense hay distintos municipios), mientras que las categorías “otras áreas metropolitanas" y "resto urbano del interior" agrupan ciudades dispersas geográficamente y con distintas dependencias políticas.

\section{Resultados}

Los tiempos diferenciales en la sala de espera como obstáculo organizacional del sistema para la consulta médica

En la tabla 1 vemos cómo los tiempos de espera en la consulta médica están asociados con el nivel educativo del entrevistado y el ingreso per cápita del hogar. Observamos que a medida que aumenta el nivel educativo, desciende el porcentaje de población que tuvo que esperar más de una hora la última vez que se atendió con un médico. Mirando los niveles extremos, vemos que seis de cada diez personas $(61 \%)$ que no terminaron la primaria debieron enfrentar este obstáculo organizacional del acceso, en comparación

2 Se incluyeron aquí a Gran Rosario, Gran Córdoba, Gran San Miguel de Tucumán y Gran Mendoza.

3 Se incluyeron aquí a Mar del Plata, Gran Salta, Gran Paraná, Gran Resistencia, Gran San Juan, Neuquén-Plottier-Cipolletti, Zárate, La Rioja, Goya, San Rafael, Comodoro Rivadavia, Ushuaia y Río Grande. 
con menos de un tercio (32\%) de los que terminaron sus estudios superiores. Algo similar sucede con el ingreso. A medida que aumenta el ingreso per cápita del hogar, desciende el porcentaje de población que ha debido esperar más de una hora en la consulta con el médico. Observando los quintiles extremos, vemos que esta situación la debió afrontar el $64 \%$ del quintil de ingreso per cápita más pobre, en contraste con el $32 \%$ del quintil de ingreso de ingreso per cápita de más dinero.

Con relación al sexo, en la tabla 1 se observa que las mujeres (49\%) debieron esperar más de una hora en mayor porcentaje que los hombres (45\%). Si bien las variables están relacionadas de forma significativa (cuando trabajamos con un $95 \%$ de confianza, pero no cuando trabajamos con un $99 \%$ ), se trata de una relación débil. En cambio, no hay diferencias significativas entre los distintos grupos etarios. Por su parte, también vemos que los tiempos de espera están fuertemente asociados con el tipo de cobertura con el que cuenta la población. Dos tercios $(64 \%)$ de quienes cuentan únicamente con la cobertura del subsector público debieron esperar más de una hora la última vez que consultaron al médico, mientras que ocurrió así solo con un $44 \%$ de quienes tienen obra social y apenas un poco más de un cuarto entre quienes tienen prepaga (28\%). Por último, vemos que es menos frecuente que sea la población que reside en la Ciudad de Buenos Aires (29\%) la que soporte esperas mayores a una hora que la del resto de los aglomerados del país. Las diferencias más importantes se observan con relación a la población residente en el Conurbano Bonaerense (54\%).

Cabe destacar que existe una relación entre las variables independientes utilizadas. $\mathrm{La}$ población que reside en la Ciudad de Buenos Aires pertenece en mayor proporción a los hogares de mayores ingresos per cápita, que
TABla 1. TiEmpo de ESPERA EN La Última CONSUlta AL MÉDICO SEGÚN CARACTERÍSTICAS SOCIOECONÓMICAS Y demográficas. Población de dieciocho años Y MÁS DE GRANDES Y MEDIANOS AGLOMERADOS DE Argentina en el año 2013 (1)

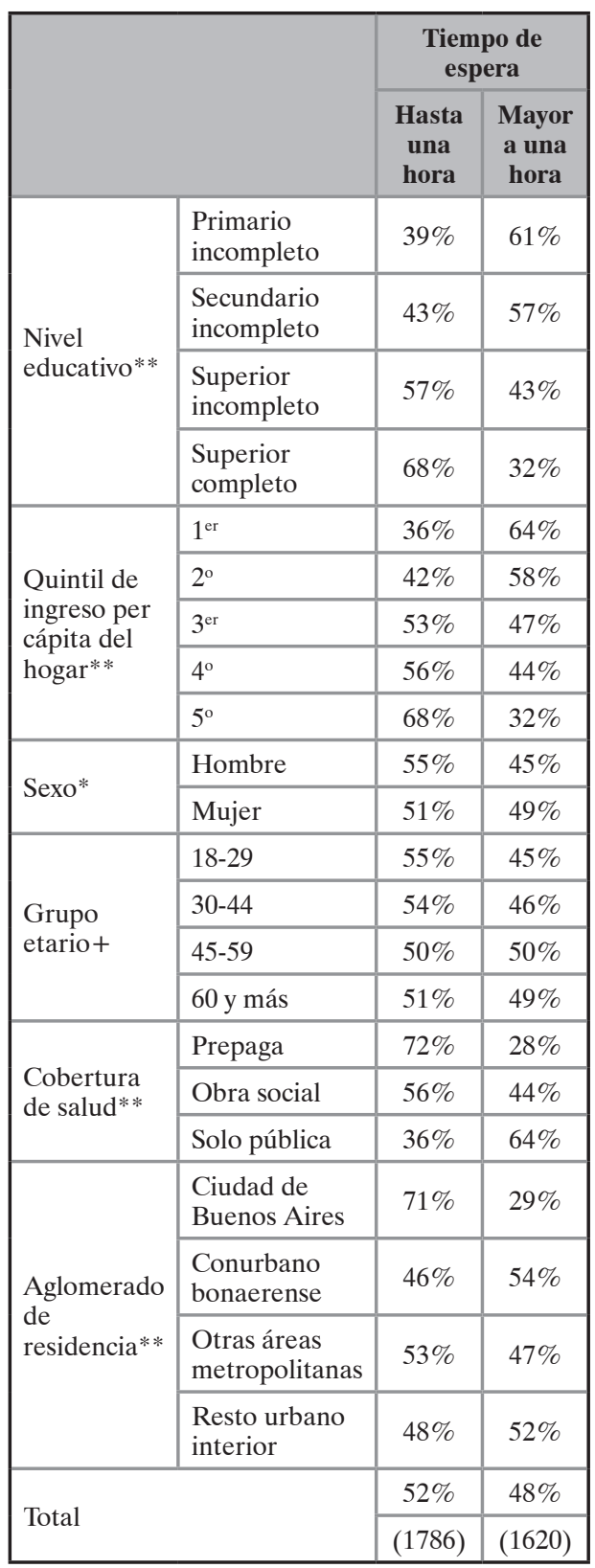

(1) No se consideró a la población que no consultó con un médico durante el último año.

Prueba chi ${ }^{2}: * \mathrm{p}<0,01,{ }^{*} \mathrm{p}<0,05,+\mathrm{p}>0,05$.

Fuente: elaboración propia con base en la EDSA 2013 
tienen mayor nivel educativo y que cuentan con cobertura de obra social o prepaga. Es más frecuente que la población de mayores niveles educativos y mayores ingresos cuente con una cobertura de obra social o prepaga, debido a que está inserta en mayor proporción en trabajos que le brindan dichas coberturas y/o tiene mayores capacidades para solventar la cobertura de su propio bolsillo. Al contar con una obra social o una prepaga, la población tiene mayor margen de acción para atenderse en establecimientos de salud por fuera del subsector público, lo que le permitiría evitarse en mayor medida las esperas prolongadas. Sin embargo, cabe preguntarse si esa es la única explicación, o si existen otros elementos que también inciden en las diferencias. Recordemos que dijimos que en el interior de las obras sociales y las prepagas existen distintos planes que ofrecen diferentes tipos de servicios. Además, también cabe destacar que las características de la atención del subsector público son muy heterogéneas en distintas zonas geográficas, debido a las desigualdades entre los recursos jurisdiccionales, pero también por distintas estrategias sanitarias locales. En este sentido, destacamos que la Ciudad de Buenos Aires es el distrito con mayores recursos humanos e infraestructura sanitaria, y uno de los que cuenta con mayor presupuesto público per cápita destinado a salud, mientras que el Conurbano Bonaerense es uno de los que menores recursos tiene $(3,5,6,38)$.

Es por ello que realizamos la regresión logística que presentamos a continuación (tabla 2). En el modelo 1 incluimos el ingreso per cápita del hogar, el máximo nivel de instrucción formal alcanzado y el sexo. No incluimos la variable grupo etario, ya que no se encontraba estadísticamente asociada. En el modelo 2 incluimos la cobertura de salud. Si la explicación de los tiempos diferenciales de espera se agotara en la cobertura, al introducir esta variable deberían dejar de ser significativos el quintil de ingreso per cápita y el nivel educativo, o bien la cobertura de salud. Por último, en el modelo 3 introducimos el aglomerado. Si los menores tiempos de espera de la población residente en la Ciudad de Buenos Aires se explicaran solo porque allí reside la población con mayores ingresos, mayor nivel educativo y que cuenta con cobertura de salud, la introducción de esta variable no tendría que resultar significativa.

En el modelo 1 vemos que tanto el ingreso como el nivel educativo están significativamente asociados con los tiempos de espera, en tanto que el sexo no lo está. Recordemos que en la lectura descriptiva vimos que el sexo tiene una asociación muy débil, por lo que es esperable este resultado. Tomando como referencia el quintil de ingreso per cápita del hogar y el nivel educativo más alto ( $5^{\circ}$ quintil y superior completo), existen diferencias significativas con el resto de los quintiles de ingreso y niveles educativos. Centrándonos en los grupos extremos, vemos que quienes alcanzaron hasta estudios primarios incompletos tienen 2,13 veces más oportunidades de esperar más de una hora que quienes tienen estudios superiores completos. En cuanto al ingreso per cápita del hogar, vemos que quienes pertenecen al quintil de menos dinero tienen 2,66 veces más probabilidades de experimentar esa demora que quienes se encuentran en el quintil que más ingreso tiene.

Cuando incorporamos la cobertura de salud (modelo 2), si bien se reducen las diferencias entre la población según su nivel educativo y su ingreso, tanto estas variables como el tipo de cobertura de salud están significativamente asociados con los tiempos de espera. Con relación al ingreso per cápita del hogar, el primer quintil pasa de tener 2,66 a 1,94 veces más oportunidades de esperar más de una hora que el quinto quintil. En cuanto al nivel educativo, la población que tiene hasta 
primaria incompleta pasa a tener 1,86 veces más oportunidades que la población con estudios superiores completos (en el modelo 1 tiene 2,13). Por su parte, la diferencia entre la población con estudios superiores y la población con estudios secundarios completos deja de ser significativa. Cabe destacar que el tipo de cobertura de salud, una vez controlado el quintil de ingreso del hogar y el nivel educativo, continúa estando significativamente asociado con los tiempos de demora en la atención. Así, vemos que quienes cuentan con una cobertura de obra social tienen 1,74 veces menos posibilidades de esperar más de una hora que los que se atienden en el sector público; mientras que quienes cuentan con una prepaga tienen 2,65 veces menos posibilidades. Un último elemento que considerar es que el ajuste del modelo mejora levemente (el R2 de Nagelkerke pasa de 0,086 a 0,106).

Por último, en el modelo 3 incorporamos también al aglomerado de residencia. Allí vemos nuevamente que si bien se reducen levemente las diferencias entre la población según su nivel educativo, su ingreso y su tipo de cobertura, continúan estando significativamente asociadas con los tiempos de espera. A diferencia del modelo anterior, dejan de

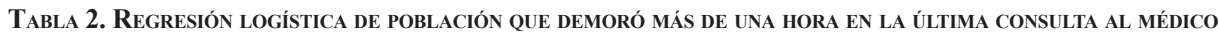
(1). Población de dieciocho años y más de grandes y Medianos aglomerados de Argentina en el año 2013

\begin{tabular}{|c|c|c|c|}
\hline & Modelo 1 & Modelo 2 & Modelo 3 \\
\hline Sexo (mujer referencia) & $0,943+$ & $0,930+$ & $0,924+$ \\
\hline Nivel educativo (superior completo referencia) & - & - & - \\
\hline Hasta primario incompleto & $2,130 * *$ & $1,859 * *$ & $1,585^{* *}$ \\
\hline Primario completo - secundario incompleto & $2,006^{* *}$ & $1,758 * *$ & $1,533^{* *}$ \\
\hline Secundario completo - superior incompleto & $1,298^{*}$ & $1,199+$ & $1,083+$ \\
\hline Quintil de ingreso per cápita del hogar ( $5^{\circ}$ referencia $)$ & - & - & - \\
\hline $1^{\text {er }}$ quintil de ingreso per cápita del hogar & $2,658 * *$ & $1,941^{* *}$ & $1,800 * *$ \\
\hline $2^{\circ}$ quintil de ingreso per cápita del hogar & $2,212^{* *}$ & $1,843^{* *}$ & $1,696 * *$ \\
\hline $3^{\text {er }}$ quintil de ingreso per cápita del hogar & $1,497^{* *}$ & $1,404 * *$ & $1,313^{*}$ \\
\hline $4^{\circ}$ quintil de ingreso per cápita del hogar & $1,371^{* *}$ & $1,328^{*}$ & $1,232+$ \\
\hline Cobertura de salud (solo pública referencia) & $\mathrm{n} / \mathrm{c}$ & - & - \\
\hline Prepaga & $\mathrm{n} / \mathrm{c}$ & $0,377 * *$ & $0,416^{* *}$ \\
\hline Obra social & $\mathrm{n} / \mathrm{c}$ & $0,576^{* *}$ & $0,603^{* *}$ \\
\hline Aglomerado (Ciudad de Buenos Aires referencia) & $\mathrm{n} / \mathrm{c}$ & $\mathrm{n} / \mathrm{c}$ & - \\
\hline Conurbano bonaerense & $\mathrm{n} / \mathrm{c}$ & $\mathrm{n} / \mathrm{c}$ & $1,579 * *$ \\
\hline Otras áreas metropolitanas & $\mathrm{n} / \mathrm{c}$ & $\mathrm{n} / \mathrm{c}$ & $1,338^{*}$ \\
\hline Resto urbano interior & $\mathrm{n} / \mathrm{c}$ & $\mathrm{n} / \mathrm{c}$ & $1,580 * *$ \\
\hline Constante & 0,392 & 0,749 & 0,602 \\
\hline R2 de Nagelkerke & 0,086 & 0,106 & 0,113 \\
\hline
\end{tabular}

Notas: (1) 0 = demoró una hora o menos; 1 = demoró más de una hora.

** $\mathrm{p}<0,01 ; * \mathrm{p}<0,05 ;+\mathrm{p}>0,05, \mathrm{n} / \mathrm{c}$ : no considerada.

Fuente: elaboración propia con base en la EDSA 2013 
ser significativas las diferencias entre los dos grupos de mayores ingresos per cápita del hogar, pero continúan siendo significativas las diferencias entre el grupo de mayores ingresos con relación a los tres de menores ingresos. Por su parte, las diferencias en los tiempos de espera entre la población residente en la Ciudad de Buenos Aires y la del resto de los aglomerados del país son significativas, aún controlado el nivel educativo, el quintil de ingreso per cápita y el tipo de cobertura. En comparación con los residentes de la Ciudad de Buenos Aires, quienes residen en el Conurbano Bonaerense y en el resto urbano tienen 1,58 veces más posibilidades de tener que aguardar más de una hora en la sala de espera al consultar al médico, mientras que los residentes en otras áreas metropolitanas tienen 1,34 veces más posibilidades. Para finalizar, cabe destacar que la incorporación del aglomerado de residencia ha generado un impacto mínimo en el grado de ajuste del modelo (el R2 de Nagelkerke pasa de $0,106$ a 0,113$)$.

Ahora bien, ¿cómo explicar que no sea solo el tipo de cobertura de salud lo que genera las diferencias entre sectores de distintos ingresos y niveles educativos en los tiempos que las personas deben esperar para la consulta con el médico? Entendemos que hay tres hipótesis complementarias para explicarlo. Por un lado, como hemos visto, dentro de un mismo subsector de atención existe una gran heterogeneidad en las características de los servicios de salud. Así, por ejemplo, es posible que los sectores de mayor ingreso y nivel educativo tengan obras sociales o prepagas que brinden mejores servicios o que residan en zonas donde el subsector público tenga mayores capacidades de atención y por lo tanto tengan que esperar menos tiempo para ser atendidos. Si bien aquí hemos controlado por el tipo de aglomerado de residencia, la variable tiene la limitación de juntar ciudades de distintas jurisdicciones, a la vez que aun en el interior de un mismo aglomerado (como el conurbano bonaerense o la Ciudad de Buenos Aires) existen diferencias entre distintas zonas en las características de los servicios (véanse 4 y 29).

Una hipótesis alternativa está vinculada con que los sectores de mayores ingresos y mayor nivel educativo tengan mayores capacidades para moverse frente a la burocracia de la organización de los servicios de salud (a partir de la comprensión de las lógicas burocrático-institucionales, las capacidades de expresión y de reclamo), y recibir un trato diferencial por parte de esta, por lo que ante un mismo servicio tardarían menos en ser atendidos. Apoya esta línea argumentativa que los sectores de menores recursos suelen tener menor conciencia sobre sus derechos a obtener servicios de calidad (que incluye la atención dentro de un tiempo adecuado), particularmente cuando estos son recibidos de forma gratuita como los del sector público, por lo que pueden ser más proclives a aceptar demoras prolongadas $(22,23)$. A ello se le agrega que la relación de los pacientes con los médicos, pero también con el personal administrativo de los centros de salud, está mediada por una relación de clase. Siguiendo a Llovet (39: 33), para los sectores estructuralmente más desfavorecidos existe una "distancia social" que hace que enfrenten "la relación sabiéndose el elemento subordinado de una asimetría”. En este sentido, esta explicación se estaría corriendo del planteamiento de Frenk (11), en tanto que el tiempo de demora en la atención no sería simplemente un obstáculo del sistema, sino que este también está relacionado con la capacidad de agencia de la población.

Una tercera hipótesis está asociada con la modalidad de atención. Como veremos en la siguiente sección, es menos frecuente que entre los sectores estructuralmente desfavorecidos se solicite un turno para acceder 
a la consulta médica. En cambio, es más frecuente que el acceso a la consulta médica se resuelva mediante la atención por servicio de urgencia (24). Debido a que los tiempos de demora en la sala de espera suelen ser más prolongados en los servicios de urgencia que en las consultas programadas, este sería otro factor que generaría que la población estructuralmente más desfavorecida deba aguardar más al momento de ser atendida.

\section{Las demoras en los turnos con médicos especialistas como obstáculo organizacional del sistema}

Como planteamos anteriormente, la demora entre la solicitud de un turno para consultar con un profesional de la salud y la atención efectiva, es otro obstáculo organizacional del sistema que puede generar una barrera en el acceso a los servicios de salud. Pero también habla de la calidad de la atención (22-25), y puede impactar en el estado de salud del paciente, ya que un tiempo de demora prolongado puede agravar la condición por la que se requiere atención (12-19). A su vez, esto puede influir en la satisfacción del paciente con relación a los servicios de salud, así como puede tener un impacto en futuras utilizaciones de los servicios de salud $(22,25,26,40)$. Entendemos que tiempos de demora demasiado prolongados entre la solicitud de un turno y la atención con el especialista puede desalentar esta modalidad de resolver la atención de la salud $(20,21)$. En este caso, trabajamos con los días de espera entre la solicitud del turno con un especialista médico y la atención efectiva por parte del especialista. El 41,7\% de la población respondió que no pidió turno con un especialista, el 5,5\% no contestó la pregunta y el 52,7\% respondió la cantidad de días que tuvo que esperar ante la última consulta con un especialista. Comenzamos trabajando con esta última población (es decir, con el $52,7 \%$ que solicitó turno con un especialista) y dicotomizamos la respuesta entre quienes tuvieron que esperar hasta un mes y entre quienes tuvieron que esperar más de un mes.

Antes de comenzar con el análisis de los datos, cabe destacar que las modalidades del acceso a la consulta con un médico especialista varían según el lugar de residencia de la población y el subsector del sistema a partir del cual accede (público, obras sociales, privado). En algunos casos, el acceso a la consulta con determinadas especialidades necesita la derivación de un médico clínico o generalista. En otros, el paciente puede solicitar directamente la consulta sin la necesidad de derivación. Ambas modalidades de acceso a la consulta con médicos especialistas están presentes tanto en el subsector público como en las obras sociales y en las prepagas (41). En el subsector público depende principalmente de las estrategias sanitarias locales, aunque en el interior de una localidad e incluso de un mismo establecimiento pueden convivir ambas modalidades $(1,34,42)$. En el subsector de las obras sociales y en el privado depende de las políticas regulatorias de las distintas entidades $(43,44)$.

En la tabla 3 puede observarse nuevamente cómo los tiempos de espera están estratificados socialmente. Allí vemos que entre quienes consultaron a un médico especialista con turno, la espera mayor a un mes fue más frecuente entre la población de menor nivel educativo, de menor ingreso, que cuenta únicamente con cobertura pública y que no reside en la Ciudad de Buenos Aires. Vemos que debió esperar más de treinta días el $28 \%$ de quienes tienen un nivel educativo hasta primario incompleto, en comparación con solo el $15 \%$ de quienes tienen estudios superiores completos. A su vez, el 25\% de la población perteneciente a los hogares de menores ingresos ( $1^{\text {er }}$ quintil) tuvo que esperar más de un mes, en contraste con solo el $13 \%$ de la población de los hogares de mayores 
ingresos per cápita ( $5^{\circ}$ quintil). También vemos que solo el $16 \%$ de la población que está afiliada a alguna cobertura de salud (ya sea obra social o prepaga) debió aguardar más de un mes para consultar al especialista médico, en tanto que fue así para el $25 \%$ de la población que cuenta únicamente con la cobertura del subsector público. Por último, puede observarse que los residentes de la Ciudad de Buenos Aires son quienes en menor medida han debido esperar más de treinta días para ser atendidos $(9 \%)$, mientras que es la población residente en otras áreas metropolitanas $(27 \%)$ la que en mayor proporción ha debido aguardar este tiempo prolongado. En cambio, no hay diferencias significativas entre hombres y mujeres, ni entre la población de distintos grupos etarios.

En la tabla 4 presentamos la regresión logística, sin introducir grupo etario ni sexo, ya que no se encuentran significativamente relacionados con la espera mayor a los treinta días entre la solicitud de turno y la atención con el médico especialista. Comenzando por el nivel educativo, vemos que las únicas diferencias significativas en la espera, tomando como referencia la población con estudios superiores completos, la encontramos con quienes tienen solo estudios primarios incompletos, una vez que controlamos la relación por el ingreso per cápita del hogar (modelo 1) y por la cobertura de salud (modelo 2). Cuando incluimos el aglomerado de residencia (modelo 3 ), las diferencias entre la población con estudios superiores y la población con primario incompleto dejan de ser significativas.

En cuanto al ingreso, en el modelo 1 vemos que una vez controlado el nivel educativo, la población perteneciente a los hogares del primer, del segundo y del tercer quintil de ingreso tiene más posibilidades, de forma 244 significativa, que la del quinto quintil de tener que esperar más de un mes entre la
Tabla 3. Días de espera para la atención CON TURNo CON UN MÉDICO ESPECIALISTA SEGÚN CARACTERÍSTICAS Socioeconómicas y demográficas. Población DE DIECIOCHO AÑOS Y MÁS DE GRANDES Y MEDIANOS aglomerados de Argentina en el año 2013 (1)

\begin{tabular}{|c|c|c|c|}
\hline & \multicolumn{2}{|c|}{$\begin{array}{c}\text { Tiempo de } \\
\text { espera }\end{array}$} \\
\hline & & $\begin{array}{c}\text { Hasta } \\
\text { un } \\
\text { mes }\end{array}$ & $\begin{array}{l}\text { Mayor } \\
\text { a un } \\
\text { mes }\end{array}$ \\
\hline \multirow{4}{*}{$\begin{array}{l}\text { Nivel } \\
\text { educativo** }\end{array}$} & $\begin{array}{l}\text { Primario } \\
\text { incompleto }\end{array}$ & $72 \%$ & $28 \%$ \\
\hline & $\begin{array}{l}\text { Secundario } \\
\text { incompleto }\end{array}$ & $79 \%$ & $21 \%$ \\
\hline & $\begin{array}{l}\text { Superior } \\
\text { incompleto }\end{array}$ & $83 \%$ & $17 \%$ \\
\hline & $\begin{array}{l}\text { Superior } \\
\text { completo }\end{array}$ & $85 \%$ & $15 \%$ \\
\hline \multirow{5}{*}{$\begin{array}{l}\text { Quintil de } \\
\text { ingreso per } \\
\text { cápita del } \\
\text { hogar** }\end{array}$} & $1^{\mathrm{er}}$ & $75 \%$ & $25 \%$ \\
\hline & $2^{\circ}$ & $76 \%$ & $24 \%$ \\
\hline & $3^{\text {er }}$ & $81 \%$ & $19 \%$ \\
\hline & $4^{\circ}$ & $85 \%$ & $15 \%$ \\
\hline & $5^{\circ}$ & $87 \%$ & $13 \%$ \\
\hline \multirow{2}{*}{ Sexo+ } & Hombre & $82 \%$ & $18 \%$ \\
\hline & Mujer & $81 \%$ & $19 \%$ \\
\hline \multirow{4}{*}{$\begin{array}{l}\text { Grupo } \\
\text { etario+ }\end{array}$} & $18-29$ & $82 \%$ & $18 \%$ \\
\hline & $30-44$ & $81 \%$ & $19 \%$ \\
\hline & $45-59$ & $80 \%$ & $20 \%$ \\
\hline & 60 y más & $82 \%$ & $18 \%$ \\
\hline \multirow{3}{*}{$\begin{array}{l}\text { Cobertura } \\
\text { de salud** }\end{array}$} & Prepaga & $84 \%$ & $16 \%$ \\
\hline & Obra social & $84 \%$ & $16 \%$ \\
\hline & Solo pública & $75 \%$ & $25 \%$ \\
\hline \multirow{4}{*}{$\begin{array}{l}\text { Aglomerado } \\
\text { de } \\
\text { residencia** }\end{array}$} & $\begin{array}{l}\text { Ciudad de } \\
\text { Buenos Aires }\end{array}$ & $91 \%$ & $9 \%$ \\
\hline & $\begin{array}{l}\text { Conurbano } \\
\text { Bonaerense }\end{array}$ & $82 \%$ & $18 \%$ \\
\hline & $\begin{array}{l}\text { Otras áreas } \\
\text { metropolitanas }\end{array}$ & $73 \%$ & $27 \%$ \\
\hline & $\begin{array}{l}\text { Resto urbano } \\
\text { interior }\end{array}$ & $81 \%$ & $19 \%$ \\
\hline \multirow{2}{*}{\multicolumn{2}{|c|}{ Total }} & $81 \%$ & $19 \%$ \\
\hline & & (2681) & $(620)$ \\
\hline
\end{tabular}

(1) No se consideró a la población que no pidió turno ni la que no respondió.

Prueba chi2: ** $\mathrm{p}<0,01,+\mathrm{p}>0,05$.

Fuente: elaboración propia con base en la EDSA 2013. 


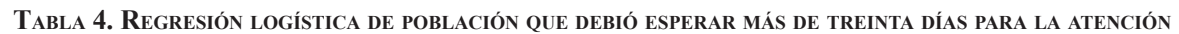
Con turno Con un médico especialista (1). Población de dieciocho años y más de gRandes y medianos aglomerados de Argentina en el año 2013

\begin{tabular}{|c|c|c|c|}
\hline & Modelo 1 & Modelo 2 & Modelo 3 \\
\hline Nivel educativo (superior completo referencia) & - & - & - \\
\hline Hasta primario incompleto & $1,513^{*}$ & $1,506^{*}$ & $1,377+$ \\
\hline Primario completo - secundario incompleto & $1,052+$ & $1,026+$ & $0,887+$ \\
\hline Secundario completo - superior incompleto & $0,907+$ & $0,889+$ & $0,794+$ \\
\hline Quintil de ingreso per cápita del hogar ( $5^{\circ}$ referencia) & - & - & - \\
\hline $1^{\text {er }}$ quintil de ingreso per cápita del hogar & $2,119 * *$ & $1,797 * *$ & $1,508^{*}$ \\
\hline $2^{\circ}$ quintil de ingreso per cápita del hogar & $2,010^{* *}$ & $1,837 * *$ & $1,513^{*}$ \\
\hline $3^{\text {er }}$ quintil de ingreso per cápita del hogar & $1,567 * *$ & $1,549 * *$ & $1,324+$ \\
\hline $4^{\circ}$ quintil de ingreso per cápita del hogar & $1,168+$ & $1,165+$ & $1,015+$ \\
\hline Cobertura de salud (solo pública referencia) & $\mathrm{n} / \mathrm{c}$ & - & - \\
\hline Prepaga & $\mathrm{n} / \mathrm{c}$ & $0,704^{* *}$ & $0,729 * *$ \\
\hline Obra social & $\mathrm{n} / \mathrm{c}$ & $0,799+$ & $0,859+$ \\
\hline Aglomerado (Ciudad de Buenos Aires referencia) & $\mathrm{n} / \mathrm{c}$ & $\mathrm{n} / \mathrm{c}$ & - \\
\hline Conurbano bonaerense & $\mathrm{n} / \mathrm{c}$ & $\mathrm{n} / \mathrm{c}$ & $1,807 * *$ \\
\hline Otras áreas metropolitanas & $\mathrm{n} / \mathrm{c}$ & $\mathrm{n} / \mathrm{c}$ & $3,098 * *$ \\
\hline Resto urbano interior & $\mathrm{n} / \mathrm{c}$ & $\mathrm{n} / \mathrm{c}$ & $1,889 * *$ \\
\hline Constante & 0,149 & 0,200 & 0,128 \\
\hline R2 de Nagelkerke & 0,030 & 0,035 & 0,060 \\
\hline
\end{tabular}

Notas: (1) $0=$ demora hasta treinta días; 1 = demora mayor a treinta días.

** $\mathrm{p}<0,01 ; * \mathrm{p}<0,05 ;+\mathrm{p}>0,05, \mathrm{n} / \mathrm{c}$ : no considerada.

Fuente: elaboración propia con base en la EDSA 2013

solicitud de un turno con un especialista médico especialista y la atención efectiva. La diferencia más importante la encontramos con la población del primer quintil, que tiene 2,12 veces más posibilidades de tener que esperar más de un mes para ser atendida. Cuando incluimos la cobertura de salud (modelo 2) y el aglomerado de residencia (modelo 3), descienden las diferencias en las oportunidades de tener que esperar más de un mes, pero continúan siendo significativas (con excepción de las diferencias con la población del tercer quintil de ingreso en el modelo 3).
Por su parte, en cuanto a la cobertura de salud observamos que la población con prepaga tiene menos posibilidades de tener que esperar más de un mes para ser atendida que la población que tiene cobertura pública, incluso una vez controlados el nivel educativo y el quintil de ingreso per cápita del hogar (modelo 2) y el aglomerado de residencia (modelo 3). En cambio, no existen diferencias significativas entre contar con una obra social y tener solo cobertura pública, una vez controlados el ingreso, el nivel educativo y el aglomerado de residencia. Por último, observamos que la población residente en los distintos grupos de aglomerados del país tiene más posibilidades de tener que aguardar 
más de un mes para ser atendida por un especialista médico que población residente en la Ciudad de Buenos Aires, incluso controlando el ingreso, el nivel educativo y la cobertura de salud. Las diferencias más importantes se encuentran con relación a la población residente en otras áreas metropolitanas, que tiene tres veces más posibilidades de tener que esperar más de treinta días que quienes residen en la Ciudad de Buenos Aires.

Por otro lado, cabe destacar que anteriormente dijimos que la EDSA permitía registrar la opción de "no pidió turno con especialista". Entendemos que este es otro punto importante que analizar, ya que puede mostrarnos cuán frecuente es esta modalidad de acceso a la consulta médica para responder a las necesidades de salud entre distintos grupos sociales. En la tabla 5 vemos que, al igual que los días de espera, la modalidad de atención de la salud mediante turnos está estratificada socialmente. Es más frecuente no haber solicitado un turno con un especialista entre la población de menor nivel educativo, perteneciente a hogares de menores recursos, que cuentan únicamente con cobertura pública y que no reside en la Ciudad de Buenos Aires. Con relación a la cobertura de salud, cabe destacar que también hay diferencias entre quienes tienen prepaga o mutual (un 27\% no solicitó turno con un médico especialista) y quienes tienen obra social (40\%). Por otro lado, existen diferencias según la edad y el sexo, de forma tal que es más frecuente que las mujeres y la población de mayor edad soliciten turnos que los hombres y la población más joven. Es posible que ello se deba a las mayores necesidades de salud de las mujeres y la población de mayor edad, pero también a que los hombres y la población más joven tienden a postergar la realización de consultas médicas $(45,46)$. zadas en el artículo (demora en la sala de
Tabla 5. Solicitud de TURNo CON UN MÉDICO ESPECIALISTA SEGÚN CARACTERÍSTICAS Socioeconómicas y demográficas. Población DE DIECIOCHO AÑOS Y MÁS DE GRANDES Y MEDIANOS aglomerados de Argentina en el año 2013 (1)

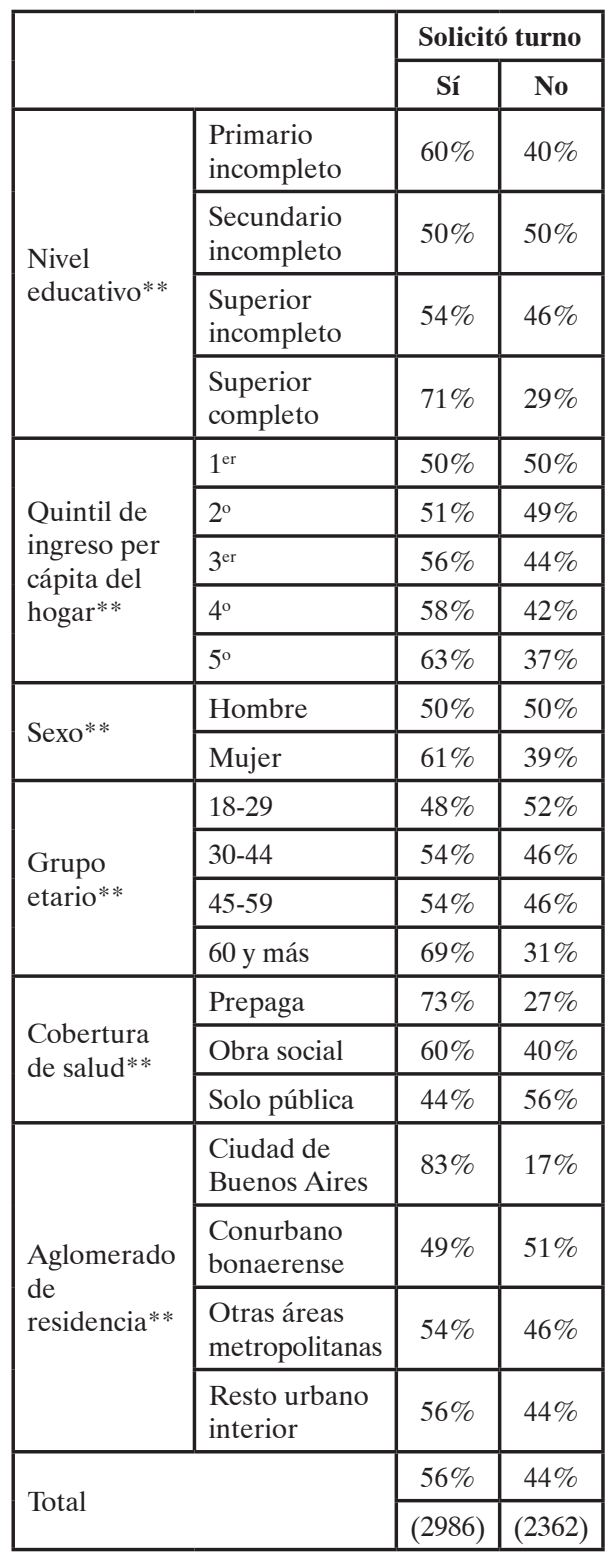

No se consideró a la población que no respondió la pregunta.

Prueba chi: ** $\mathrm{p}<0,01$.

Fuente: elaboración propia con base en la EDSA 2013. 
espera, demora en los turnos con médicos especialistas y la atención mediante un turno con médicos especialistas) dan cuentan de elementos complementarios, que en parte muestran distintos aspectos interrelacionados de un mismo fenómenos. Los sectores estructuralmente más desfavorecidos tienen mayores demoras entre que solicitan un turno con un médico especialista y reciben la atención. Ello puede ser uno de los motivos por los cuales es menos frecuente que utilicen esta modalidad de atención para dar respuesta a sus necesidades de salud, y sea más frecuente que recurran a los servicios de urgencia médica (24). Debido a que las esperas en los servicios de urgencia suelen ser mayores que las consultas con turno programadas, este podría ser uno de los factores por los cuales la población de menores niveles educativos e ingresos, que cuenta únicamente con cobertura pública y que no reside en la Ciudad de Buenos Aires, debe aguardar más tiempo en la sala de espera cuando consulta a un médico.

Destacamos que los días de espera entre la solicitud del turno con un médico especialista y la atención efectiva no es el único obstáculo organizacional por el cual el sistema de salud argentino dificulta más a la población de menos recursos acceder a la consulta programada con un médico especialista. Distintos estudios $(30,31,34)$ han señalado que en determinados establecimientos del subsector público los trámites asociados a la obtención de los turnos, así como los horarios limitados de atención dificultan el acceso a estos.

\section{Conclusión y discusión}

Son los grupos sociales estructuralmente más desfavorecidos (de menor ingreso, menor nivel educativo y que cuentan únicamente con cobertura pública) los que en mayor medida deben aguardar más de una hora en la sala de espera para ser atendidos por un médico y quienes más deben esperar entre la solicitud de un turno con un especialista médico y la atención efectiva. Es decir, es a la población que menos recursos para cuidar la salud tiene, entre ellos tiempo disponible y capacidad de planificación de su vida cotidiana a mediano plazo (47), la que el sistema de salud argentino le hace esperar más para ser atendida. Además, estas diferencias varían según la localización geográfica de la población. Los tiempos de espera se reducen para la población residente en la Ciudad de Buenos Aires (el aglomerado del país con mayor infraestructura física y recursos humanos y con uno de los mayores presupuestos de salud per cápita) y tienden a acrecentarse en otros aglomerados urbanos. Las diferencias entre la población de distintos ingresos y niveles educativos en la demora mayor a un mes en los turnos con médicos especialistas, tienden a desaparecer cuando controlamos por aglomerado de residencia y tipo de cobertura de salud. En cambio, disminuyen pero continúan siendo significativas las diferencias entre la población con distintos niveles educativos e ingresos en las demoras en la sala de espera en la consulta médica, aun controlando el aglomerado de residencia y el tipo de cobertura de salud.

Además, también hemos visto que son los grupos estructuralmente más desfavorecidos los que con menor frecuencia solicitan turno para consultar con un especialista médico. Es posible que la no solicitud de turno como modalidad de atención de la salud esté en parte influenciada por las percepciones diferenciales de los procesos de salud-enfermedad-atención, teniendo en cuenta los distintos tiempos y lugares que ocupa el cuidado de la salud en la vida cotidiana de la población $(39,47-49)$. Sin embargo, también entendemos que es posible que entre los sectores estructuralmente más desfavorecidos, la modalidad de solicitar un 
turno con un especialista sea una opción menos viable para resolver las necesidades de atención de su salud, teniendo en cuenta que tienen que esperar más para ser atendidos que otros sectores. Experiencias previas (propias o de conocidos) de espera demasiado prolongadas para la atención a partir de turnos con especialistas pueden generar que para estos sectores la opción más viable para intentar resolver sus necesidades de salud sea recurrir directamente al servicio de urgencia médica (24) y que en algunos casos se busquen otras alternativas y/o se retrase la consulta médica hasta situaciones límites, "cuando el deterioro es tal que amerita la "urgencia"' (21: 10). Destacamos que otros obstáculos organizacionales asociados con la obtención de los turnos (necesidad de ir al establecimiento previamente para obtener el turno, en horarios específicos, esperas prolongadas), así como la oferta de turnos en una franja horaria limitada, también contribuyen a que para los sectores con menores recursos sea más viable la atención a partir de los servicios de urgencia.

Debido a que las esperas en los servicios de urgencia suelen ser mayores que las consultas con turno programadas, este podría ser uno de los factores por los cuales la población de menores niveles educativos e ingresos, que cuenta únicamente con cobertura pública y que no reside en la Ciudad de Buenos Aires debe aguardar más tiempo en la sala de espera cuando consulta a un médico. En este sentido, creemos que los tiempos de espera diferenciales son un elemento que puede generar percepciones diferenciales sobre procesos de salud-enfermedad-atención, haciendo que para algunos sectores sociales la atención mediante la solicitud de un turno sea particularmente lejana en su horizonte de posibilidades. res como la población adulta mayor, a pesar de tener tiempos de espera similares a los hombres y a la población más joven, son los que de forma más frecuente solicitan turno con especialistas. Entendemos que ello está asociado a necesidades diferenciales de utilizar los diversos servicios (en este caso consultar con distintos especialistas médicos), pero también a factores culturales que generan que sea más frecuente que los hombres, los sectores de menor ingreso y nivel educativo, y la población más joven tienda a postergar la realización de consultas médicas $(45,46)$.

Por último, destacamos que nosotros hemos analizado los tiempos de espera (en el establecimiento y por turnos) como una forma a partir de la cual el sistema de salud genera obstáculos diferenciales para realizar una consulta médica. Distintos antecedentes han dado cuenta de cómo ello tiene consecuencias sobre el acceso a los servicios de salud (27-34). También hemos planteado que los tiempos diferenciales en la espera hacen a la calidad de los servicios de salud y pueden impactar en la percepción de los proceso de salud-enfermedad-atención. Pero además, esta temática puede ser vista como una forma de estratificación social en sí misma. Los tiempos de espera en las distintas facetas de la vida son diferenciales en función del lugar ocupado en la estructura social. Como sostiene Schwartz, "rigen relaciones típicas entre la posición del individuo dentro de un sistema social y la medida que espera y es esperado por otros miembros del sistema" (50:46). Así, a quien le hacen esperar un tiempo inusualmente largo, como el caso de los pacientes para ser atendidos por un médico, lo hacen ser sujeto "de una afirmación que dice que nuestro tiempo (y por lo tanto, nuestro valor social) vale menos que el tiempo de quien impone la espera y que tenemos menos mérito que esa persona" (50: 46). En este sentido, nos interesa destacar que la espera, desde la perspectiva de la población y particularmente en el campo de 
la medicina, puede resultar un padecimiento en sí mismo y está asociado con experiencias que generan angustias e impotencia $(50,51)$.

\section{Agradecimientos}

Se agradece a Agustín Salvia y a Cecilia Tinoboras por facilitar el acceso a los datos de la Encuesta de la Deuda Social Argentina, desarrollada por el Observatorio de la Deuda Social Argentina de la Universidad Católica Argentina. También a Betina Freidin y Ruth Sautu por los aportes realizados al artículo.

\section{Referencias bibliográficas}

1. Chiara M, Di Virgilio MM, Moro J. Inequidad (es) en la atención de la salud en el Gran Buenos Aires, Argentina. Una mirada desde la gestión local. Postdata. 2009 Ene.-Jul.; 14 (1): 97-128.

2. Maceira D, Olaviaga S, Kremer P, Cejas C. Centros de Atención Primaria de Salud: radiografía de su distribución en la Argentina. Medicina y Sociedad. 2010 Dic.; 30 (4): 1-14.

3. Programa de las Naciones Unidas para el Desarrollo (PNUD). El sistema de salud argentino y su trayectoria de largo plazo: logros alcanzados y desafíos futuros. Aportes para el Desarrollo Humano en Argentina. Buenos Aires: PNUd; 2011.

4. Lago FP, Elorza ME, Moscoso SN, Ripari NV. Equidad en el acceso a los servicios de Atención Primaria de Salud en sistemas de salud descentralizados: el caso de la provincia de Buenos Aires, Argentina. Rev Gerenc Polit Salud. 2013 Jul.Dic.; 12 (25): 40-54.

5. Cetrángolo O. Financiamiento fragmentado, cobertura desigual y falta de equidad en el sistema de salud argentino. Revista de Economía Política de Buenos Aires. 2014 Nov.; 13 (8): 145-82.

6. Maceira D. Descentralización y equidad en el Sistema de Salud Argentino. Buenos Aires: Cedes; 2008.

7. Báscolo E. Cambios de los mercados de salud en la seguridad social en Argentina. Santiago de Chile: Cepal; 2008 [Internet] [acceso: 2 de diciembre de 2015]. Disponible en: http://www.eclac.cl/cgi-bin/ getProd.asp? xml=/publicaciones/xml/5/35135/ P35135.xml\&xsl=/ues/tpl/p9f.xsl\&base $=/$ ues/tpl/ top-bottom.xsl.

8. Findling L, Arruñada M, Klimovsky E. La desregulación de obras sociales: un balance de la libre elección. En La salud en crisis. Buenos Aires: Editorial Dunken; 2000. p. 55-67.

9. Fidalgo M. Adiós al derecho a la salud. El desarrollo de la medicina prepaga. Buenos Aires: Espacio; 2008.
10. Maceira D. Inequidad en el acceso a la salud en la Argentina. Buenos Aires: CIPPEC; 2008.

11. Frenk J. El concepto y medición de la accesibilidad. Salud Publica Mex. 1985 Sep.-Oct.; 27 (5): 438-53.

12. Ramos S, Pantelides EA. Dificultades en la prevención secundaria del cáncer de cuello de útero: las mujeres y los médicos frente a una citología cervical de resultado positivo. Buenos Aires: Cedes; 1990.

13. Wiesner C, Tovar S, Cendales R, Vejarano M. Organización de los servicios de salud para el control del cáncer de cuello uterino en el municipio de Soacha. Rev Colomb Cancerol. 2006; 10 (2): 98-108.

14. Sociedad Argentina de Cardiología. Realidad del manejo del infarto agudo de miocardio con elevación del segmento st en la Argentina. Rev Argent Cardiol. 2008 Mayo-Jun.; 76 (3): 226-8.

15. Fernández Concepción O, Buergo Zuaznabar M. Necesidad de cambios organizativos en el Sistema Nacional de Salud para la atención del ictus. Revista Cubana de Salud Pública. 2008 Abr.-Jun.; 34 (2).

16. Piombo AC, Rolandi, F, Fitz Maurice M, Salzberg S, Strumminger M, Zylberstejn H, et al. Registro de calidad de atención del infarto agudo de miocardio en los hospitales públicos de la ciudad de Buenos Aires. Revista Argentina de Cardiología. 2011 Mar.-Abr.; 79 (2): 133-8.

17. Piñeros M, Sánchez R, Perry F, García OA, Ocampo R, Cendales R. Demoras en el diagnóstico y tratamiento de mujeres con cáncer de mama en Bogotá, Colombia. Salud Pública de México. 2011 Nov.-Dic.; 53 (6): 478-85.

18. Rodríguez-Angulo E, Palma-Solís M, ZapataVázquez R. Causas de demora en la atención de pacientes con complicaciones obstétricas ¿qué es necesario atender? Ginecol Obstet Mex. 2014 Oct.; 82: 647-58.

19. Camejo C, Legman C, Gaye A, Arcieri B, Brumett F, Castro L, et al. Unidad de ACV en el Hospital de Clínicas: comportamiento clínico-epidemiológico de los pacientes con ACV (2007-2012). Archivos de Medicina Interna. 2015 Mar.; 37 (1): 30-5.

20. Gutiérrez MA, Gogna M, Caromero M. Estudio de caso. Programas de salud reproductiva para adolescentes en Buenos Aires, Argentina. En Gogna M, coordinadora. Programas de salud reproductiva para adolescentes. Buenos Aires: Cedes; 2001.

21. Lorenzetti M. Relaciones interétnicas y prácticas de atención de la salud en el Chaco Salteño. Corpus. Archivos virtuales de la alteridad americana. 2011 Jul.-Dic.; 1 (2): 1-15.

22. Petracci M, Ramos S, Romero M. Calidad de la atención: perspectivas de las mujeres usuarias de los servicios de prevención de cáncer cérvico uterino. Buenos Aires: Cedes; 2007 [Internet] [acceso: 18 de enero de 2016]. Disponible en: http://www. cedes.org.ar/Publicaciones/IF/2001/1847.pdf.

23. Cerrutti M, Freidin B. Evaluando la calidad de la atención en servicios de planificación familiar en la ciudad de Buenos Aires: medidas estandarizadas y percepción de las usuarias. Buenos Aires: Cenep; 2004

24. Otero R. Oferta, demanda y calidad de atención 249 médica. Revista del ITAES. 2006; 8 (4): 9-13. 
25. Velandia Salazar F, Ardón Centeno N, Jara Navarro MI. Satisfacción y calidad: análisis de la equivalencia o no de los términos. Rev Gerenc Polit Salud. 2007 Jul.-Dic.; 6 (13): 139-68.

26. Díaz Grajales C, Zapata Bermúdez Y, Aristizábal Grisales JC. Acceso y satisfacción con servicios curativos: análisis de casos en afiliados al régimen contributivo y afiliados al régimen subsidiado en un barrio estrato 2 - Cali, Colombia. Rev Gerenc Polit Salud. 2015 Ene.-Jun.; 14 (29): 155-78.

27. Stolkiner A, Comes Y, Parenti M, Solitario R, Unamuno P. Descentralización y equidad en salud: Estudio sobre la utilización de servicios de salud de la Ciudad de Buenos Aires por parte de usuarios pobres del conurbano bonaerense. Buenos Aires: Cedes; 2003.

28. Findling L, Abramzón M, Peterlini C. Desigualdades en salud: de la teoría a los indicadores. Actas del II Congreso Nacional de Sociología ¿Para qué la sociología en la Argentina actual?; 2004 Oct 20-23; Buenos Aires, Argentina. Buenos Aires: Carrera de Sociología, UBA; 2004.

29. Báscolo E, Yavich N, Urquía, M. Capacidades locales de gestión como factor predictivo de la utilización y accesibilidad a los servicios de primer nivel para la obtención de cuidados prenatales. Rev Gerenc Polit Salud. 2007 Ene.-Jun.; 6 (12) 112-25.

30. Checa S, Erbaro C, Schvartzman E, Tapia S. Calidad de la atención de la salud sexual y reproductiva para adolescentes desde la perspectiva de género y derechos en servicios de salud adolescente de efectores públicos de CABA. Actas de las IX Jornadas Nacionales de debate interdisciplinario en salud y población; 2011 Ago. 10-12; Buenos Aires, Argentina. Buenos Aires: Instituto de Investigaciones Gino Germani; 2011.

31. Maceira D, Urrutia M, Olaviaga S, Cerrizuela B, Chami C, Díaz A, et al. Necesidades y acceso a los servicios de salud de la población adolescente en el norte argentino. Rev. Argentina de Salud Pública. 2014 Sep.; 5 (20): 17-24.

32. Ponce M. El cuidado de la salud de los que cuidan. Las cuidadoras familiares. En López E, Findling L, coordinadoras. De cuidados y cuidadoras. Buenos Aires: Biblos; 2015.

33. Ballesteros MS. Freidin B. Reflexiones sobre la conceptualización y la medición del acceso a los servicios de salud en Argentina: el caso de la Encuesta Nacional de Factores de Riesgo 2009. Salud Colectiva. 2015 Oct.-Dic.; 11 (4): 523-35.

34. Chiara M, Moro J, Ariovich A, Jiménez C. La política sanitaria local en el Municipio de Malvinas. Los Polvorines: UNGs; 2010 [Internet] [acceso: 18 de enero de 2016]. Disponible en http://www.urbared.ungs. edu.ar/pdf/pdf-salud/DT_DGPS_MA_set2011web.pdf.

35. Quartulli D, Tinoboras C, Vera J, De Grande P. Anexo metodológico. La encuesta de la deuda social argentina del bicentenario (2010-2016). En: Salvia A, editor. Estado de situación del desarrollo humano y social: Barreras estructurales y dualidades de la sociedad argentina en primer año del Bicentenario. Buenos Aires: Educa; 2011.
36. Salvia A, Adaszko, D. Anexo metodológico. En: Salvia A, editor. Un régimen consolidado de bienestar con desigualdades sociales persistentes. Claroscuros en el desarrollo humano y la integración social (2010-2013). Buenos Aires: Educa; 2014.

37. Sánchez Vizcaíno G. Regresión logística. En: Luque Martínez T, coordinador. Técnicas de análisis de datos en investigación de mercados. Madrid: Ediciones Pirámide; 2000.

38. Tobar F, Montiel L, Falbo R, Drake I. La Red Pública de Atención Primaria de la Salud en Argentina. Documento de Difusión. 2006 [Internet] [acceso: 15 de diciembre de 2015]. Disponible en: http://www. federicotobar.com.ar/nf_pdf5/La_Red_Publica.pdf.

39. Llovet JJ. Servicios de salud y sectores populares. Los años del proceso. Buenos Aires: Cedes; 1984.

40. Mendoza-Sassi R, Beria JU. Utilización de los servicios de salud: una revisión sistemática de los factores relacionados. Cadernos de Saúde Pública. 2001 Jul.-Ago.; 17 (4): 819-32.

41. Stolkiner A, Comes Y, Garbus P. Alcances y potencialidades de la Atención Primaria de la Salud en Argentina. Ciência \& Saúde Coletiva. 2011 Jun.; 16(6): 2807-16.

42. Gómez SA, de Paepe P, Conde LL, Stolkiner A. La implementación local de la atención primaria en la Argentina: estudio de caso en el municipio de Lanús, provincia de Buenos Aires. Saúde em Debate. 2012 Jul.-Sep.; 36 (94): 436-48.

43. Espelt C. La APs desde la perspectiva de una obra social. En: Torres R, editor. Atención Primaria de la Salud. Nuevas Dimensiones. Buenos Aires: ISALUd; 2001. p. 98-111.

44. Dillon H. El Gatekeeper, ¿una necesidad imprescindible para el equilibrio de los sistemas? Una visión. En: Torres R, editor. Atención Primaria de la Salud. Nuevas dimensiones. Buenos Aires: ISALUD; 2001. p. 138-45.

45. Kornblit AL. Culturas juveniles. Buenos Aires: Eudeba; 1997.

46. Freidin B, Krause M. La atención de la salud y la percepción de riesgos: género, ciclo vital, y experiencias biográficas. En: Freidin B, coordinadora. El cuidado de la salud: mandatos culturales, experiencias biográficas y prácticas cotidianas en sectores medios de Buenos Aires. Buenos Aires: Imago Mundi; 2016.

47. Boltanski L. Los usos sociales del cuerpo. Buenos Aires: Periferia; 1974.

48. Menéndez E. De sujetos, saberes y estructuras: introducción al enfoque relacional en el estudio de la salud colectiva. Buenos Aires: Lugar; 2009.

49. Phelan, JC, Link, BG, Tehranifar P. Social conditions as fundamental causes of health inequalities: theory, evidence, and policy implications. Journal of Health and Social Behavior. 2010; 51(1 supl.): 128-40.

50. Auyero J. Pacientes del Estado. Buenos Aires: Eudeba; 2013.

51. Scribano A. Primero hay que saber sufrir...!!! Hacia una sociología de la espera como mecanismo de soportabilidad social. En Scribano A, Lisdero P, compiladores. Sensibilidades en juego: miradas múltiples desde los estudios sociales de los cuerpos y las emociones. Córdoba: CEA-Conicet; 2010. 